\title{
Handling CCI and ICI in OFDMA Femtocell Networks Through Frequency Scheduling
}

\author{
Mustafa E. Şahin, Ismail Guvenc, Member, IEEE, Moo-Ryong Jeong, Member, IEEE, \\ and Hüseyin Arslan, Senior Member, IEEE
}

\begin{abstract}
Femtocells have a strong potential for increasing the efficiency and coverage of next-generation broadband wireless networks. In this paper, a co-channel framework for the coexistence of orthogonal frequency division multiple access (OFDMA) based macrocell and femtocell wireless networks is proposed. It is based on utilizing the resource blocks of macrocell-associated mobile stations ( $m M S s$ ) that are far away to a femtocell base station $(f B S)$, therefore avoiding strong interference that may occur between a femtocell and close-by mMSs. An avoidance method that jointly utilizes the spectrum sensing results as well as scheduling information obtained from the macrocell base station (mBS) is introduced. Moreover, the impact of intercarrier interference (ICI) from the mMSs in the uplink is discussed and evaluated through simulations.
\end{abstract}

Index Terms - Femtocell, OFDMA, co-channel interference, inter-carrier interference, cognitive radio.

\section{INTRODUCTION}

The demand for broadband wireless data rates has been pushing the wireless technology to new horizons. The data rate requirements for the next-generation wireless networks have been specified under the standard referred as the IMTAdvanced, where, peak rates on the order of 1 Gbps are targeted for low-mobility scenarios. Femtocells, which have been recently introduced as a new class of personal-use base stations (BSs) [1]-[3], can help to achieve some of the key requirements of the IMT-Advanced standard. For example, they can improve the peak data rates by more than 40 times and the mean data rates by 200 times according to an analysis in [4].

One of the important benefits of femtocells is the elimination of coverage area problems for indoor scenarios. Some other benefits of femtocells include reduced capital and operational expenditure, reduced bandwidth load and power requirements, increased average revenue per user, and deployment in operator-owned spectrum. As illustrated in Fig. 1 , a femtocell BS (fBS) is connected to the mobile operator's core network through existing broadband Internet connection of the user. A macrocell-associated mobile station (mMS) does not have to be a dual mode terminal in order to communicate

M. E. Sahin and H. Arslan are with the Electrical Engineering Department, University of South Florida, Tampa, FL 33620, USA (e-mail: msahin@mail.usf.edu; arslan@eng.usf.edu).

I. Guvenc and M. R. Jeong are with DOCOMO Communications Laboratories USA, Inc., Palo Alto, CA 94304, USA (e-mail: \{iguvenc, jeong\}@docomolabs-usa.com). both with an fBS and a macrocell BS (mBS) ${ }^{1}$.Besides their uses for enhancing communications, femtocells can interact with smart home appliances and personal computers inside the house. This way, an fBS-centric network of home electronics can be formed, which would enable the users to remotely connect to any electrical device at their homes as shown in Fig. 1. The reader is referred to [5] for an in depth discussion of controlling home appliances in a smart home environment.

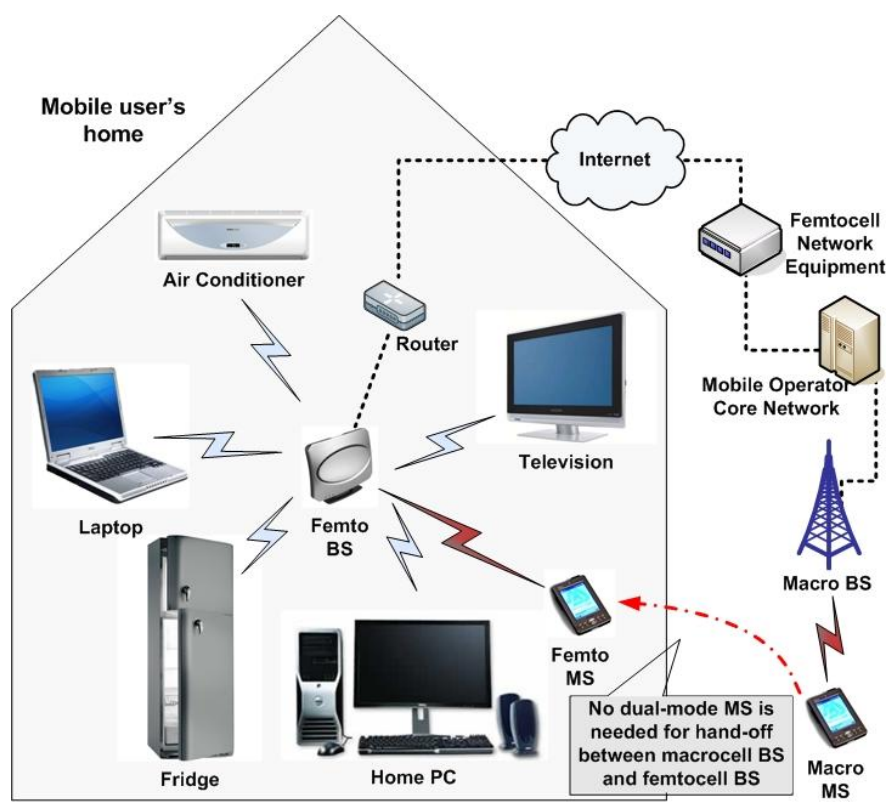

Fig. 1 Femtocell deployment at homes. Connection between the macrocell and femtocell is established through the Internet. Home appliances can be controlled by the user through the fBS.

Femto Forum, which is a business organization established in July 2007 to promote femtocell deployments, provides some femtocell applications in [6] that might be attractive to consumers. In these exemplary applications, femtocell enables users to

- Automatically synchronize their music collection on their mobile device with their home PC when a they return home,

- Control their TV from their mobile device,

- Leave virtual fridge notes to be delivered when the recipient returns home,

- Remotely control home appliances such as air conditioning when out of the house.

${ }^{1}$ Note that the abbreviations H-UE, HNB, M-UE, and NB are used in 3GPP documents for referring to femtocell mobile station (fMS), fBS, mMS, and $\mathrm{mBS}$, respectively. 
Femtocell networks have been studied extensively under the 3GPP standard (see e.g., [7], [8], and the references therein), where wideband code division multiple access (W-CDMA) is used as the physical layer technology. Even though system capacity and performance have been analyzed for macrocells and femtocells in the presence of co-channel interference (CCI) in [1], [3], [9], it can be said that these studies are more specific to CDMA based systems. There are relatively limited studies on orthogonal frequency division multiple access (OFDMA) based femtocell networks, which offer greater flexibility in terms of allocation of frequency resources. In this work, the focus is on the next-generation OFDMA based cochannel femtocells, which share the same spectrum with the macrocell network and aim an efficient utilization of spectrum resources while causing minimum interference to the macrocell network. Some related work on avoiding interference in OFDMA networks through spectrum sensing [10] and through intelligent radio resource allocation [11], [12] is available in the literature in the context of cognitive radio systems; however, these work do not consider systemspecific issues related to femtocells.

The major goal in this paper is to introduce an interference avoidance framework between a femtocell and the mMSs, which is based on not using the resource blocks occupied by closely located mMSs. Availability of macrocell frequency scheduling information is considered, and this information is effectively utilized in conjunction with spectrum sensing. Moreover, inter-carrier interference (ICI) from macrocell UL to the femtocell UL is analyzed. The variation of ICI with respect to mMS-to-fBS distance is investigated via simulations, and how ICI affects the decisions about UL spectrum opportunities at a femtocell is demonstrated.

The rest of the paper is organized as follows. The system model is provided in Section II. CCI and ICI problems in the co-channel femtocell deployment are discussed in Section III. In Section IV, the proposed CCI avoidance framework is introduced. Simulation results are demonstrated in Section V, and the last section concludes the paper.

\section{SYSTEM MODEL}

Since both the macrocell and femtocell networks considered are OFDMA based, their signals can be modeled in the same way. Also, since the downlink signal has a model that is similar to any of the uplink user signals (except for the frequency scheduling), only the UL signal model will be given. Consider an OFDMA system with $\mathrm{N}_{\mathrm{u}}$ users in the UL. The sampled time domain signal at the transmitter of user $i$ can be written as

$$
\begin{array}{r}
x_{i}(n)=\sqrt{P_{\mathrm{tx}, i}} \sum_{k \in \Gamma_{i}} X_{i}(k) \exp \quad j 2 \pi k n / N, \\
-N_{\mathrm{CP}} \leq n \leq N-1,
\end{array}
$$

where $P_{t x, i}$ is the transmit power for user $i, k \in \boldsymbol{\Gamma}_{i}$ is the subcarrier index, $\Gamma_{i}$ is the set of subcarriers of length $N_{i}$ assigned to user $i$ out of $N$ total subcarriers, $N_{\mathrm{CP}}$ is the length of the cyclic prefix $(\mathrm{CP})$, and $X_{i}(k)$ is the data on the $k$ th subcarrier $i$ th user.

The time domain aggregate received signal is the superposition of signals from all users, each of which propagates through a different multipath channel and arrives at the receiver with a delay $\delta_{i}=\left[N \tau_{i} / T\right]$, where $\tau_{i}$ is the propagation delay experienced by user $i$, and $T$ is the duration of the useful part of the symbol. Then, aggregate discrete-time received signal can be expressed as

$$
y(n)=\sum_{i=1}^{N_{\mathrm{u}}} y_{i}(n)+\omega(n)
$$

where $\omega(n)$ denotes the additive white Gaussian noise $(\mathrm{AWGN})$, and

$$
\begin{aligned}
y_{i}(n)= & \sqrt{P_{\mathrm{rx}, i}} \sum_{l=0}^{L-1} \alpha_{i}(l) \\
& \times \sum_{m=-\infty}^{\infty} x_{i}^{(m)} n-D_{l, i}-m\left(N+N_{\mathrm{CP}}\right),
\end{aligned}
$$

where $m$ is the symbol index, $P_{\mathrm{rx}, i}$ is the received power for user $i, L$ denotes the total number of multipath components (MPCs), $\alpha_{i}(l)$ is the $l$ th MPC for user $i$, and $D_{l, i}=\left\lceil N \tau_{l, i} / T\right\rceil+\delta_{i}$, where $\tau_{l, i}$ is the delay of the $l$ th MPC for user $i$. If $D_{l, i} \leq N_{\mathrm{CP}}$, the frequency domain signal for the $k$ th subcarrier of user $i$ is given by

$Y_{i}^{(m)}(k)=\sqrt{E_{\mathrm{sc}, i}} X_{i}^{(m)}(k) \sum_{l=0}^{L-1} \alpha_{i}(l) \exp -j 2 \pi k D_{l, i} / N$

where $E_{\mathrm{sc}, i}$ is the average received energy per subcarrier for user $i$, which is equal to $P_{\mathrm{rx}, i}$.

\section{CCI AND ICI ISSUES IN FEMTOCELL DEPLOYMENTS}

\section{A. Deployment Configurations}

There are two common spectrum access approaches for femtocell deployments: 1) Dedicated channel access, and 2) Co-channel access. For dedicated channel operation, femtocells are assigned a separate channel than that of the macrocell. Even though this approach mostly eliminates potential interference from the macrocell, frequency resources are not efficiently utilized. Co-channel deployment enables more efficient utilization of the available spectrum, where all the frequency resources of the macrocell are re-used only by the few users of the femtocell. Co-channel deployments have been commonly preferred in the prior-art works on femtocell networks due to more efficient utilization of the spectrum resources (see e.g., [1], [9], [13]). For example, [1] reports simulation results with co-channel femtocell access, where the median of the system-wide sum-throughput improves by nearly $250 \mathrm{~b} / \mathrm{s} / \mathrm{Hz}$ with respect to a scenario without femtocells. However, co-channel interference still remains a critical problem, especially for scenarios where there are mMSs in the vicinity of a femtocell. In the access mode referred as the closed-subscriber-group (CSG) mode [7], close-by mMSs are 
not allowed to make hand-off to the femtocell network. Especially for femtocells on the edge of the macrocell, this implies significant interference concerns between the mMSs and the femtocell. One of the contributions of the present work is a method for handling the interference from close-by mMSs, by avoiding using their frequency resources at the femtocell network, which will be discussed in more detail in Section IV.

\section{B. CCI Scenarios for $\mathrm{mMSs}$}

As discussed in the previous subsection, co-channel implementation brings the advantage of efficient spectrum usage. However, it also results in CCI between the femtocell(s) and the macrocell in various ways. In Fig. 2, different CCI possibilities are listed according to their sources, their targets, and whether they occur in the DL or the UL.

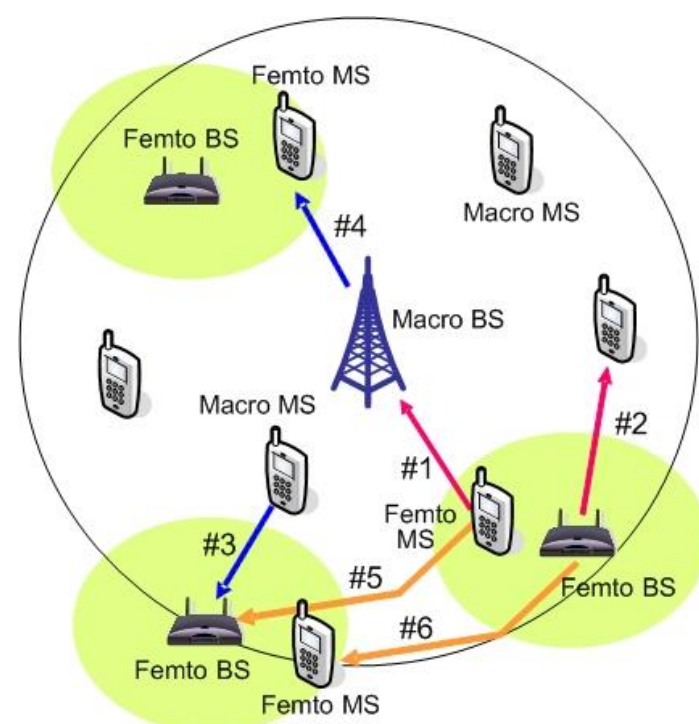

\begin{tabular}{|c|c|c|c|}
\hline & \multicolumn{2}{|c|}{ Interference } & \multirow{2}{*}{ UL- } \\
\cline { 1 - 2 } & From & To & DL \\
\hline$\# 1$ & Femto MS & Macro BS & UL \\
\hline$\# 2$ & Femto BS & Macro MS & DL \\
\hline$\# 3$ & Macro MS & Femto BS & UL \\
\hline$\# 4$ & Macro BS & Femto MS & DL \\
\hline$\# 5$ & Femto MS & $\begin{array}{l}\text { Other } \\
\text { Femto BS }\end{array}$ & UL \\
\hline \#6 & Femto BS & $\begin{array}{l}\text { Other } \\
\text { Femto MS }\end{array}$ & DL \\
\hline
\end{tabular}

Fig. 2 All possible interference scenarios related to femtocell communications.

Interference scenarios \#1 - \#2 involve the CCI caused by the femtocell network to the macrocell network, scenarios \#3 \#4 involve the CCI caused by the macrocell network to the femtocell network, and scenarios \#5-\#6 are the CCI scenarios between close-by femtocell networks. All these interference scenarios can be considered for both time division duplex (TDD) and frequency division duplex (FDD) systems. It should be noted that these scenarios are based on the rule that femtocell is not allowed to be in DL while macrocell is in UL (in TDD systems), or femtocell cannot use the UL frequency band of the macrocell for DL (in FDD systems). In this paper, we consider scenario \#3, which is the interference to an fBS due to the UL transmission of macrocell MSs, as well as \#4, which is the interference from the mBS to the fMSs.

The distance between an mMS and a femtocell network may be as large as twice the cell radius. This suggests that even with co-channel operation, CCI between a femtocell network and some far away mMSs may be insignificant. Hence, both in UL and DL, a femtocell network may intelligently utilize the resource blocks of these far away mMSs, and avoid co-channel operation with close-by mMSs in order to minimize the CCI problem. On the other hand, since $\mathrm{mBS}$ is located at the center of the macrocell, its distance to any particular femtocell is smaller than the cell radius. Hence, the received interference by the femtocell is considerably strong and it needs to be cancelled at the fMSs. Details of addressing the CCI will be presented in Section IV.

\section{ICI in the Uplink}

A different type of interference that may be observed in next generation femtocell deployments is the inter-carrierinterference (ICI) [1], [14], [15]. It is specific to the uplink and it is caused by the timing misalignment of mMS signals at the femtocell BS. Typically, the mMSs get synchronized to the $\mathrm{mBS}$ in the uplink through a ranging process, where the $\mathrm{mBS}$ determines and announces the instant to start transmission for each mMS. Since such a synchronization mechanism is not applicable for the fBS, the mMS signals arrive at the fBS with different delays due to their different distances to the fBS, as illustrated in Fig. 3(b).

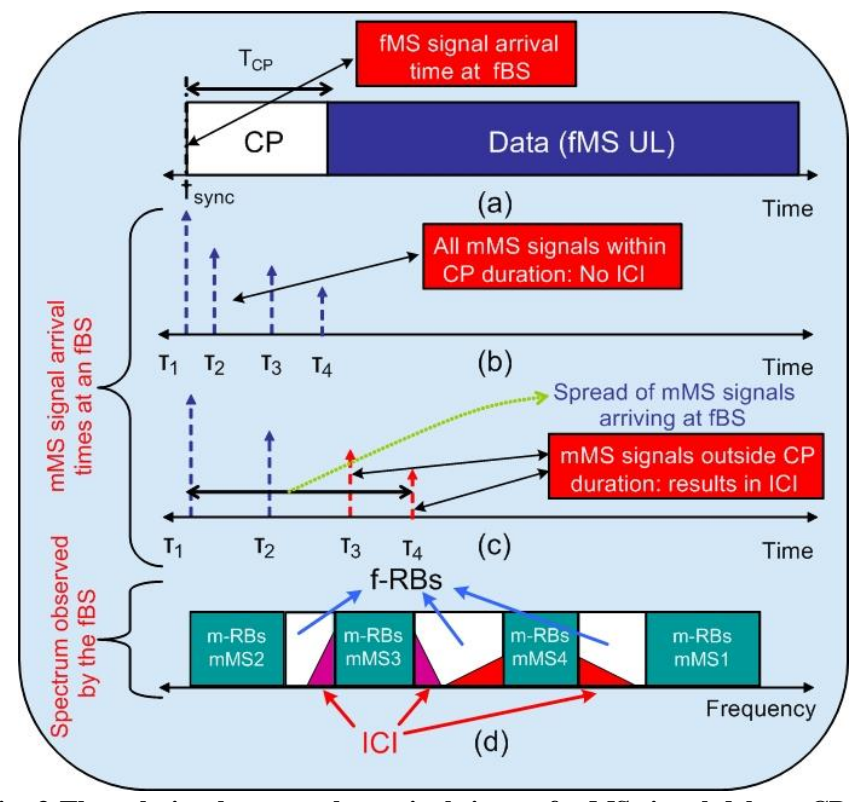

Fig. 3 The relation between the arrival times of mMS signal delays, CPsize, and ICI. (a) Structure of a femtocell symbol, (b) Signal arrival times from four different mMSs at an fBS that are within the CP, (c) mMS signal arrival times that exceed the CP, and (d) Illustration of ICI due to delays larger than the femtocell $\mathrm{CP}$ size. (m-RB: Macrocell resource block, f-RB: Femtocell resource block)

Assuming that the femtocell is synchronized to the first arriving mMS signal in the uplink, if the delays of some of the 
other mMSs exceed the CP of the femtocell symbol (see Fig. 3(c)), orthogonality between the subcarriers is lost. This causes inter-carrier interference in the resource blocks that can be reused by the femtocell network, as illustrated in Fig. 3(d). This is especially a critical problem for femtocells that are located closer to the macrocell edge [15] due to the increased spread of the mMS arrival times.

When there are some $D_{l, i}>N_{\mathrm{CP}}$, the received signal on the $k$ th subcarrier of user $i$ can be written by getting the FFT of (3) as follows

$$
\begin{aligned}
& Y_{i}^{(m)}(k)=\frac{\sqrt{E_{\mathrm{sc}, i}}}{N} \sum_{l=0}^{L-1} \alpha_{i}(l) \\
& \times\left\{\sum_{n=0}^{D_{l, i}-N_{\mathrm{CP}}-1} x_{i}^{(m-1)}\left(n+N+N_{\mathrm{CP}}-D_{l, i}\right) \exp -j 2 \pi k n / N\right. \\
& \left.+\sum_{n=D_{l, i}-N_{\mathrm{CP}}}^{N-1} x_{i}^{(m)}\left(n-D_{l, i}\right) \exp -j 2 \pi k n / N\right\}
\end{aligned}
$$

After plugging (1) into (5) and some manipulation, we have

$$
Y_{i}^{(m)}(k)=\sqrt{E_{\mathrm{sc}, i}} \sum_{l=0}^{L-1} \alpha_{i}(l) S_{i, l}(k)+I_{i, l}(k),
$$

where the desired signal and the total ICI are respectively given as

$$
\begin{gathered}
S_{i, l}(k)=X_{i}^{(m)}(k) K_{i, l}(k) \exp -j 2 \pi k D_{l, i} / N \\
I_{i, l}(k)=\frac{1}{N} \sum_{\substack{p \in \Gamma_{i} \\
p \neq k}}\left[\frac{1-\exp -j 2 \pi(p-k)\left(D_{l, i}-N_{\mathrm{CP}}\right) / N}{1-\exp -j 2 \pi(p-k) / N}\right] \\
\times-X_{i}^{(m)}(p) \exp -j 2 \pi p D_{l, i} / N \\
+X_{i}^{(m-1)}(p) \exp j 2 \pi p\left(N_{\mathrm{CP}}-D_{l, i}\right) / N
\end{gathered}
$$

where

$$
K_{i, l}(k)=\frac{N-\left(D_{l, i}-N_{\mathrm{CP}}\right)}{N} \times \mathrm{I}(i, k),
$$

with $\mathrm{I}(i, k)$ denoting an indicator function given by

$$
\mathrm{I}(i, k)= \begin{cases}1, & \text { if } k \in \Gamma_{i}, \\ 0, & \text { if } k \notin \Gamma_{i} .\end{cases}
$$

The user distance $\left(d_{i}\right)$ has two opposing effects on the ICI power. Since user delays are directly proportional to $d_{i}$ through $D_{1, i}=d_{i} /\left(\mathrm{c} T_{\mathrm{s}}\right)$, where $\mathrm{c}$ is the speed of light, and $T_{\mathrm{s}}$ is the sampling time, a longer distance leads to a larger delay and hence a stronger ICI. On the other hand, since $E_{\mathrm{sc}, i}$ also depends on $d_{i}$ through $\mathrm{E}_{\mathrm{sc}, i}=P_{t x, i} \lambda^{2} /\left[(4 \pi)^{2} d_{i}{ }^{\gamma}\right]$, where $\lambda$ is the wavelength of the transmitted signal, and $\gamma$ is the path loss exponent, weaker ICI should be observed at large distances. Therefore, as will also be verified through simulations in Section V, ICI power in (8) is expected to be maximized at a certain distance and then will start decreasing with $d_{i}$.

Knowledge about the variation of received signal as well as ICI powers with respect to distance can be especially useful in the following specific scenario. Assume that the macrocell BS provides the fBSs not only with the mMS scheduling information but also with the geographical coordinates of the mMSs. Moreover, the fBSs are not capable of spectrum sensing, which means that, for determining the spectrum opportunities, they solely depend on the information that they receive from the $\mathrm{mBS}$. In this scenario, the $\mathrm{fBS}$ can determine the usable occupied resource blocks according to the received signal power versus distance information. The unoccupied resource blocks that should be avoided due to ICI, on the other hand, can be found according to the ICI versus distance information.

\section{HANDLING CCI AND ICI THROUGH FREQUENCY SCHEDULING}

\section{A. General Framework}

In a CSG femtocell deployment, the potential CCI problem between the femtocell and mMSs both in UL and DL can be prevented by ensuring that femtocell avoids using the macrocell resource blocks that belong to nearby mMSs as illustrated in Fig. 4 in a simple way.
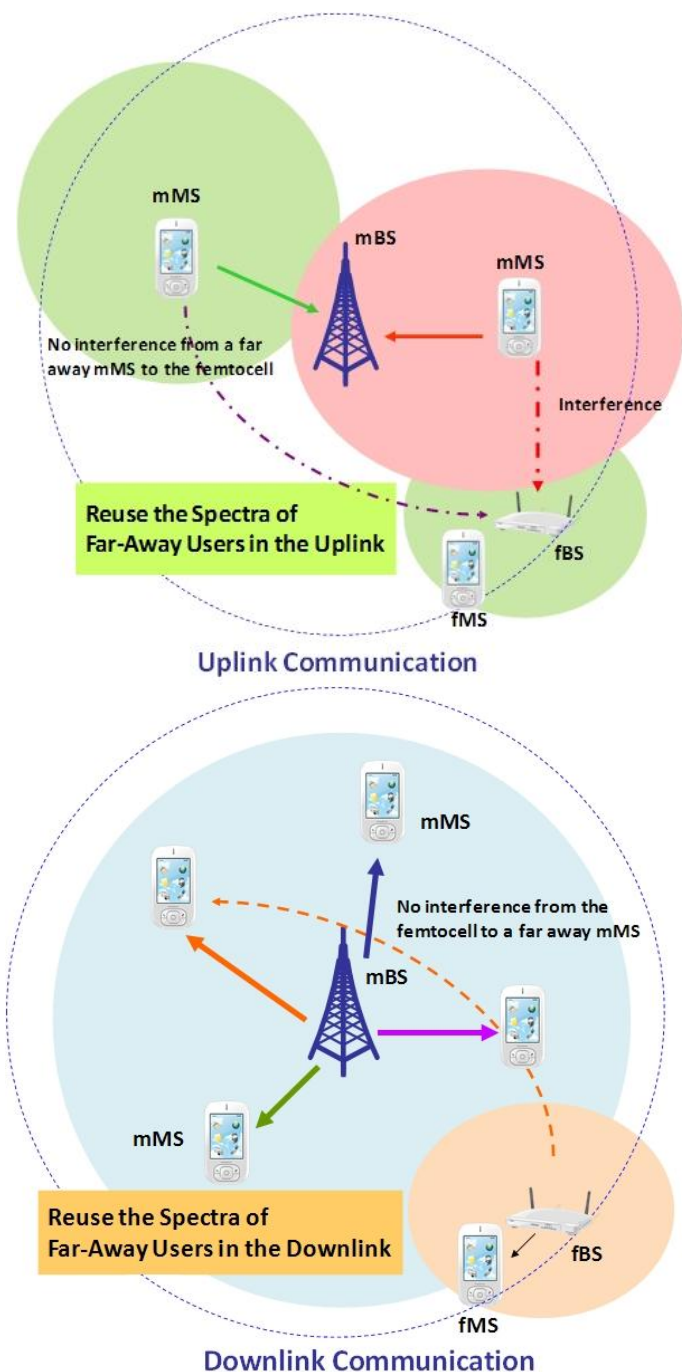

Fig. 4 Framework for handling the interference between femtocell and macrocell in the downlink and in the uplink. Femtocell senses the spectrum during UL, identifies the close-by users through UL scheduling information, and avoids using their spectrum specified in DL scheduling information. 
The steps of the CCI avoidance based framework that we propose for the UL can be summarized as follows. First, the fBS receives the $\mathrm{mMS}$ scheduling information from the $\mathrm{mBS}$. Then, it performs spectrum sensing for finding the occupied parts of the spectrum, which are supposedly the resource blocks of nearby mMSs and determines the spectrum opportunities by comparing the sensing results with the scheduling information. Finally, the fBS schedules its fMSs over the spectrum opportunities determined.

The main points of handling the CCI in the downlink, on the other hand, can be summarized as follows:

- In the uplink, fBS determines the occupied UL resource blocks through joint utilization of scheduling information and spectrum sensing results (see Section IV.C for more details). Sources of high signal power are labeled as closely located users.

- The UL scheduling information indicates which resource blocks belong to which user. Hence, this information can be used to individually identify each closely located user.

- Utilizing the DL scheduling information, again obtained from the $\mathrm{mBS}$, the DL resource blocks that belong to the identified closely located users are found.

- fBS avoids using the resource blocks of close-by users not to cause any interference to them in the DL. The interference from the $\mathrm{mBS}$ that hits the fMSs, on the other hand, is cancelled via advanced cancellation methods by the fMSs.

An example for the CCI cancellation methods that can be employed by the fMSs is introduced in [16], where a Least Mean Square (LMS) Maximum Likelihood Estimation (MLE) method is proposed to estimate the time varying channels and the transmitted symbols simultaneously. Also, an MLE based joint demodulation algorithm is provided in [17] for a receiver with multiple antennas where availability of reliable channel estimates is assumed. A detailed discussion of these cancellation methods, however, is beyond the scope of this paper. In the remainder of this section, two critical steps of the proposed co-channel femtocell operation framework will be discussed.

\section{B. Obtaining Scheduling Information from the $m B S$}

We consider two possible methods for the fBSs to obtain the scheduling information of mMSs from the mBS. These two methods are receiving the scheduling information through the backhaul, and capturing the scheduling information over-theair. In the first method, upon initialization, fBS establishes a secure and stable backhaul connection to the operator network and obtains information about the mMS resource blocks. The $\mathrm{mBS}$ needs to make and then deliver the scheduling decision well ahead of the scheduled transmission, the ahead time being greater than the latency of the backhaul. In the second method, the mMS scheduling information is received from the $\mathrm{mBS}$ over the air. For this purpose, fBS may connect to the $\mathrm{mBS}$ as an $\mathrm{mMS}$, and use this connection in order to obtain the scheduling information.

\section{Jointly Utilizing Scheduling Information and Spectrum \\ Sensing Results}

In a well designed OFDMA system, it is expected that almost all resource blocks are allocated to users. Therefore, scheduling information that the fBS obtains from the $\mathrm{mBS}$ would normally indicate that the spectrum is mostly occupied. However, since many of the mMSs are far away from the fBS, their resource blocks can still be utilized at the femtocell. Through spectrum sensing, an fBS can detect resource blocks that are either not used by the macrocell network, or belong to far away users.

The occupied parts of the UL spectrum can be found by simple means such as energy detection. Energy detection is performed by taking the Fourier transform of the received time domain signal $r(t)$, which yields a $1 \times \mathrm{N}$ frequency-domain sample vector $\mathbf{r}_{\text {rec }}$. Then, its magnitude squared is computed, and compared with an energy detection threshold $\varepsilon_{\text {thrs }}^{(\mathrm{ED})}$. Energy detection outputs for all the received subcarriers can be written as

$$
\Gamma_{\mathrm{ED}}=\mathrm{U}\left|\mathbf{r}_{\mathrm{rec}}\right|^{2}-\varepsilon_{\mathrm{thrs}}^{(\mathrm{ED})},
$$

where $\mathrm{U}(\mathrm{x})$ denotes the unit step function that individually applies to all the elements of a vector $\mathrm{x}$, and $\Gamma_{\mathrm{ED}}$ is a $1 \times \mathrm{N}$ vector with elements $\in\{0,1\}$ (a 0 implies that the subcarrier is sensed as unoccupied, while a 1 implies that the subcarrier is sensed as occupied).

Once the spectrum sensing results are available, the fBS may compare the spectrum sensing results with the UL scheduling information to decide about the spectrum opportunities. Denoting the $1 \times \mathrm{N}$ sized scheduling vector of an $\mathrm{mMS}-j$ with $\Gamma_{\text {sch }}^{(j)}$ (elements of the scheduling vector are 0 s and $1 \mathrm{~s}$ ), the decision for occupied subcarriers can be made as follows.

$$
\Gamma_{\mathrm{used}}=\sum_{j=1}^{N \mathrm{u}}\left\{\mathrm{U}\left(\frac{\left\langle\Gamma_{\mathrm{ED}}, \Gamma_{\mathrm{sch}}^{(j)}\right\rangle}{\left\|\Gamma_{\mathrm{sch}}^{(j)}\right\|_{1}}-\varepsilon_{\mathrm{jnt}}\right)\right\} \times \Gamma_{\mathrm{sch}}^{(j)},
$$

where $\langle x, y\rangle$ is the inner product of vectors $\mathrm{x}$ and $\mathrm{y},\|\mathrm{x}\|_{1}$ is the L1-norm of a vector $\mathrm{x}$, and $0<\varepsilon_{\text {jnt }}<1$ is a joint detection threshold. For example, if $\varepsilon_{\text {jnt }}=0.5$, this means that at least half of the sub-carriers from a scheduling vector need to be sensed as occupied so that the mMS is identified as a nearby mMS. An appropriate value for $\varepsilon_{\text {jnt }}$ determined through simulations is 0.75 .

Spectrum sensing results are impaired with missed detections (MD) and false alarms (FA) due to additive noise. In the macrocell uplink, another reason for these impairments is the ICI that is caused by the timing misalignment. 


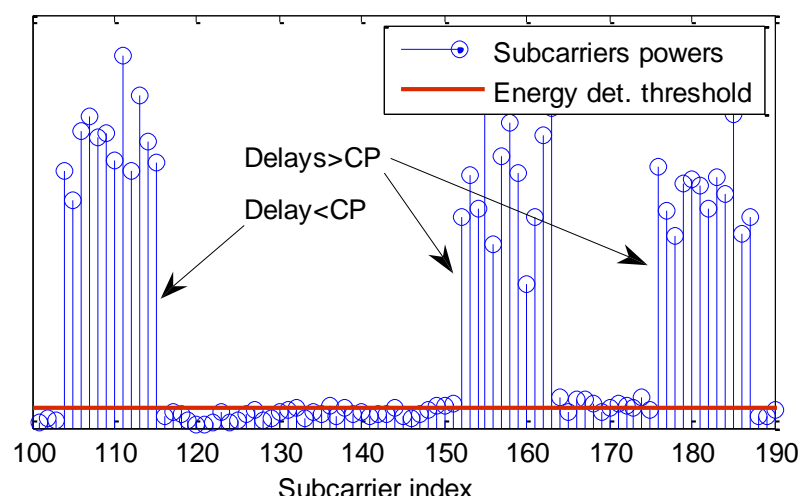

(a)

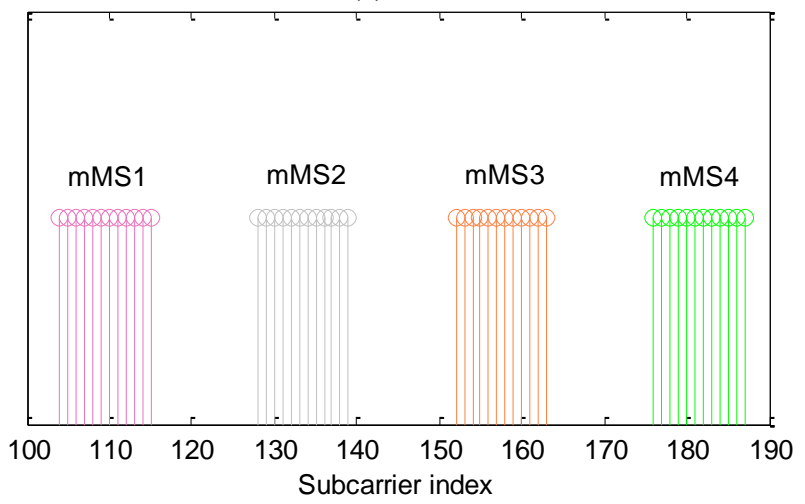

(b)

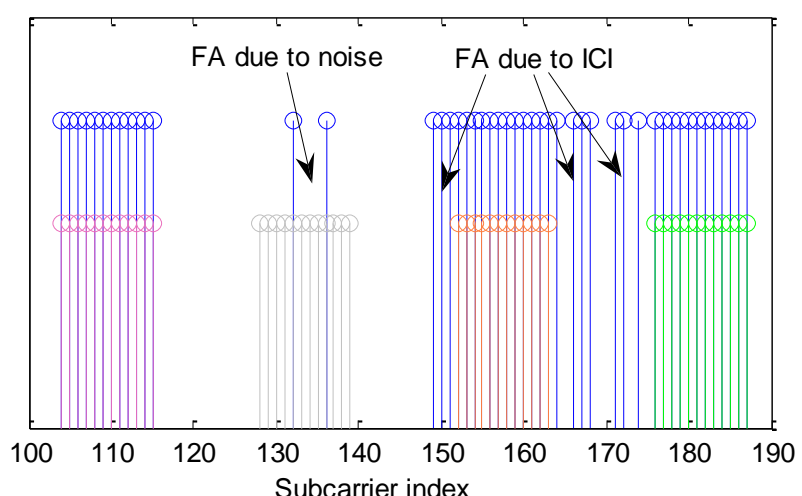

(c)

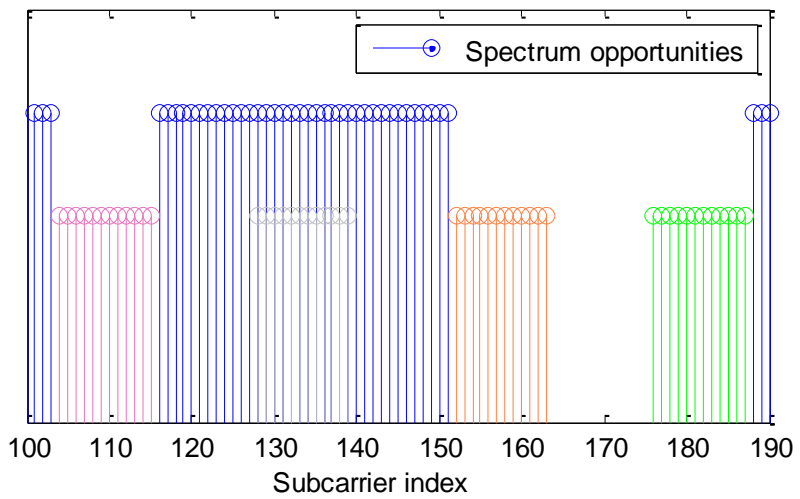

(d)

Fig. 5 Combining scheduling information with spectrum sensing results. (a) Received subcarrier powers and the energy detection threshold, (b) Scheduling information for multiple mMSs, where an LTE resource block size of 12 subcarriers is considered. (c) Energy detection results matched with scheduling information, false alarms occur due to noise and due to ICI, (d) Spectrum opportunities detected.

If the interference level of mMS- $j$ is strong, it satisfies

$$
\mathrm{U}\left\langle\Gamma_{\mathrm{ED}}, \Gamma_{\mathrm{sch}}^{(j)}\right\rangle /\left\|\Gamma_{\mathrm{sch}}^{(j)}\right\|_{1}-\varepsilon_{\mathrm{jnt}}=1 .
$$

This implies that if that mMS's delay is larger than the CP of a femtocell signal, it may also yield a strong ICI in some resource blocks leading to false alarms. Denote $\boldsymbol{\Pi}_{\text {int }}$ as the set of mMSs that satisfy the above equality. In order to detect if there are any resource blocks that are subject to strong ICI, the femtocell checks the neighboring resource blocks of mMSs that belong to the set $\boldsymbol{\Pi}_{\text {int }}$. Typically, ICI may impact only few resource blocks within the neighborhood of a certain subcarrier. Letting $\eta_{\text {ICI }}$ denote the number of subcarriers where ICI may be considered as dominant, the set of subcarriers that will be checked for the presence of ICI is given by the following vector

$$
\begin{aligned}
& \Gamma_{\mathrm{sch}, 2}\left(\eta_{\mathrm{ICI}}\right)= \\
& \sum_{j \in \Pi_{\mathrm{int}}}\left(\operatorname{sign}\left[\sum_{k=-\eta_{\mathrm{ICI}}}^{k=\eta_{\text {III }}} \Lambda_{\mathrm{shift}}\left(\Gamma_{\mathrm{sch}}^{(j)}, k\right)\right]-\Gamma_{\mathrm{sch}}^{(j)}\right),
\end{aligned}
$$

where $\Lambda_{\text {shift }}(\mathrm{x}, k)$ is a logical right-shift operator which shifts the elements of a bit-vector $\mathrm{x}$ in $k$ bit positions towards right, and $\operatorname{sign}(\mathrm{x})$ is a sign operator which operates individually on all the elements of a vector $\mathrm{x}$. Once the subcarriers that may be subject to ICI are obtained in vector $\Gamma_{\text {sch,2 }}\left(\eta_{\text {ICI }}\right)$, they are compared with the energy detection threshold $\varepsilon_{\mathrm{thrs}}^{(\mathrm{ED})}$ as follows

$$
\Gamma_{\mathrm{ICI}}=\mathrm{U} \Gamma_{\mathrm{sch}, 2}\left(\eta_{\mathrm{ICI}}\right) \otimes\left|\mathbf{r}_{\mathrm{rec}}\right|^{2}-\varepsilon_{\mathrm{thrs}}^{(\mathrm{ED})}
$$

where $\otimes$ is used to indicate a term-by-term product. Then, all the occupied resource blocks are captured by the sum $\Gamma_{\text {ICI }}+\Gamma_{\text {used }}$, whose complementary set yields the spectrum opportunities. Note that if any information about the delays from the mMSs are available, such information can be utilized in obtaining the set $\boldsymbol{\Pi}_{\text {int }}$, where mMSs whose delays are within the CP-length are not included into the set since they do not cause significant ICI (they may still yield some ICI due to their multipath components).

As a simple example, consider the macrocell UL scenario illustrated in Fig. 5, where a resource block size of 12 subcarriers as in the Long Term Evolution (LTE) standard is considered. The fBS compares the spectrum sensing results (shown in Fig. 5(c)) with the scheduling information (shown in Fig. 5(b)) to determine the resource blocks that constitute spectrum opportunities. Energy detection applied to the 
received signal (Fig. 5(a)) yields some FAs due to noise and due to ICI as shown in Fig. 5(c). These false alarms may or may not cause a resource block to be considered as occupied based on their number within each resource block, e.g. if the $\varepsilon_{\text {jnt }}$ is 0.75 , the FAs need to exceed $25 \%$ of the block size for the block to be detected as occupied. The spectrum opportunities found for the illustrated scenario are shown in Fig. 5(d), where scheduling information is plotted again for visual comparison. Note that the spectrum sensing results indicate that the received signal power for mMS2 is relatively weak because it is apparently a far-away mMS. This means that the resource blocks associated with mMS2 may be utilized by the femtocell both in the uplink and in the downlink. Moreover, the resource block located around subcarriers 170 cannot be used even though the scheduling information indicates that it is available. This is due to the strong ICI from the subcarriers of mMS-3 and mMS-4.

\section{Simulation Results}

Computer simulations are performed to analyze the variation of ICI with respect to distance as well as to quantify the effect of ICI on the successful detection of empty parts of the spectrum. The path loss model used in the simulations, which is derived from the Okumura-Hata model, is obtained from [8]. The model, which is applicable to small to medium cities, yields the path loss (in $\mathrm{dB}$ ) as follows

$$
\begin{aligned}
L=46.3 & +33.9 \log \left(f_{\mathrm{c}}\right)-13.82 \log \left(h_{\mathrm{b}}\right) \\
& +44.9-6.55 \log \left(h_{\mathrm{b}}\right) \log (d)-F\left(h_{\mathrm{M}}\right),
\end{aligned}
$$

where $f_{\mathrm{c}}$ is the center frequency, $h_{\mathrm{b}}$ is the base station height above ground, $h_{\mathrm{M}}$ is the mobile station height above ground, and $F\left(h_{\mathrm{M}}\right)$ is given by

$$
F h_{\mathrm{M}}=1.1 \log \left(f_{\mathrm{c}}\right)-0.7 h_{\mathrm{M}}-1.56 \log \left(f_{\mathrm{c}}\right)-0.8 \text {. }
$$

Concerning the scenario at hand, $h_{\mathrm{b}}$ should be considered as the height of a femtocell BS, which is found in a high-rise building. The parameters related to wave propagation and path loss used in the simulations are mainly selected according to the values given in [3] and [8]. All simulation parameters are listed in Table I.

TABLE I

SiMULATION PARAMETERS

\begin{tabular}{ll}
\hline \hline Parameter & Value \\
\hline Center frequency (fc ) & $700 \mathrm{MHz}, 2 \mathrm{GHz}$ \\
Bandwidth (B) & $5.714 \mathrm{MHz}$ \\
FFT size & 512 \\
Symbol duration & $89.9 \mu \mathrm{sec}$ \\
CP sizes & $1 / 32,1 / 16,1 / 8,1 / 4$ \\
BS height (hb ) & $30 \mathrm{~m}, 50 \mathrm{~m}$ \\
MS height (hM ) & $2 \mathrm{~m}$ \\
MS transmit power & $27 \mathrm{dBm}$ \\
Antenna gain & $16 \mathrm{~dB}$ \\
Wall penetration loss & $15 \mathrm{~dB}$ \\
Number of Walls & $1(\mathrm{external})$ \\
Noise floor of fBS & $-174 \mathrm{dBm} / \mathrm{Hz}+10 \log 10(\mathrm{~B})$ \\
& $=-106.43 \mathrm{dBm}$ \\
\hline
\end{tabular}

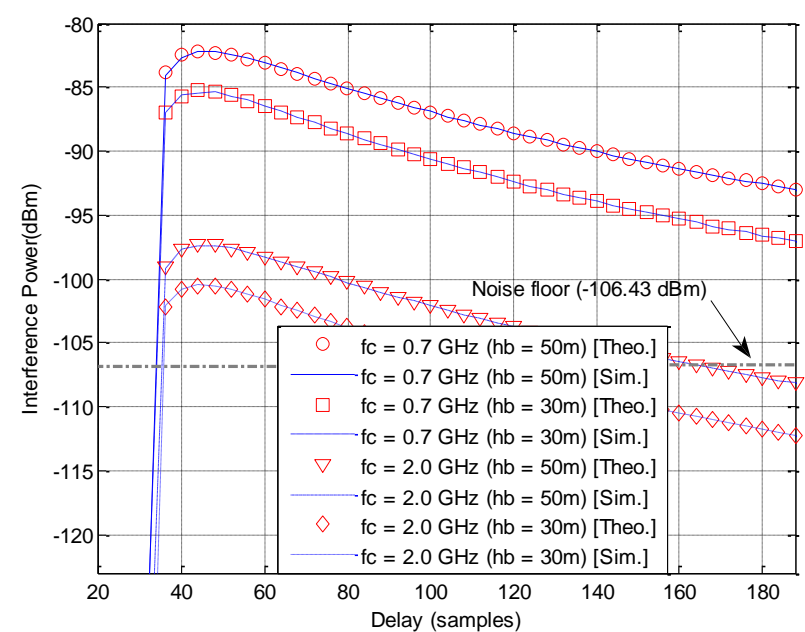

Fig. 6 Variation of ICI power wrt. RTD for different center frequencies and femtocell BS heights.

The variation of the ICI power depending on the round-tripdelay (RTD) is plotted in Fig. 6, where the largest delay corresponds to a distance of $5 \mathrm{~km}$. To obtain the simulated results, the resource blocks allocated to the mMS2 are randomly spread around the given spectrum, and ICI is measured by determining the total energy in the unused resource blocks. The theoretical curves, on the other hand, are plotted utilizing the expressions for ICI given in (8) to verify the simulation results. From the curves plotted, it can be concluded that

- ICI is typically close to zero for delays smaller than the $\mathrm{CP}$ size. Once the $\mathrm{CP}$ is exceeded, there is a sharp rise in the ICI, but at larger distances ICI decreases due to increased path loss.

- Under certain conditions, ICI power might be lower than the noise floor, i.e. the effect of ICI can become negligible.

To investigate how the ICI power is affected by the changes in certain important system parameters, the ICI versus delay analysis is performed for two different center frequencies and two BS heights (employing a CP size of 32 samples). The results demonstrated in Fig.6 show that employing a lower center frequency or having the BS at a higher location might considerably increase the received interference power.

Another analysis is performed on the error probability in detecting the occupied and unoccupied subcarriers in the received UL signal via energy detection. The error probability is computed as the sum of probability of missed detection (PMD) and probability of false alarm (PFA). PMD is defined as the ratio of number of subcarriers detected as unused although they are used to the total number of subcarriers $\mathrm{N}$. PFA, on the other hand, is the ratio of the number of subcarriers detected as used although they are unused to $\mathrm{N}$.

Two different subcarrier assignment schemes are considered. The first one is a blockwise assignment, where the two schemes used in the simulations are an LTE resource block with 12 subcarriers and 7 symbols, and a WiMAX UL PUSC 
tile with 4 subcarriers and 3 symbols. These schemes will be shortly denoted as $12 \times 7$ and $4 \times 3$, respectively. The other assignment scheme considered is a randomized assignment, where each individual subcarrier may be assigned to a different user. The two randomized assignment schemes employed in the simulations are $1 \times 7$ and $1 \times 3$. The maximum RTD that the latest arriving user signal can have $\left(\tau_{\max }\right)$ is considered to be between $0 \mu \mathrm{s}$ and $60 \mu \mathrm{s}$, where all other user RTDs are between $0 \mu$ s and $\tau_{\max }$. Note that $\tau_{\max }$ values greater than $11.2 \mu$ s exceed the $\mathrm{CP}$ duration.

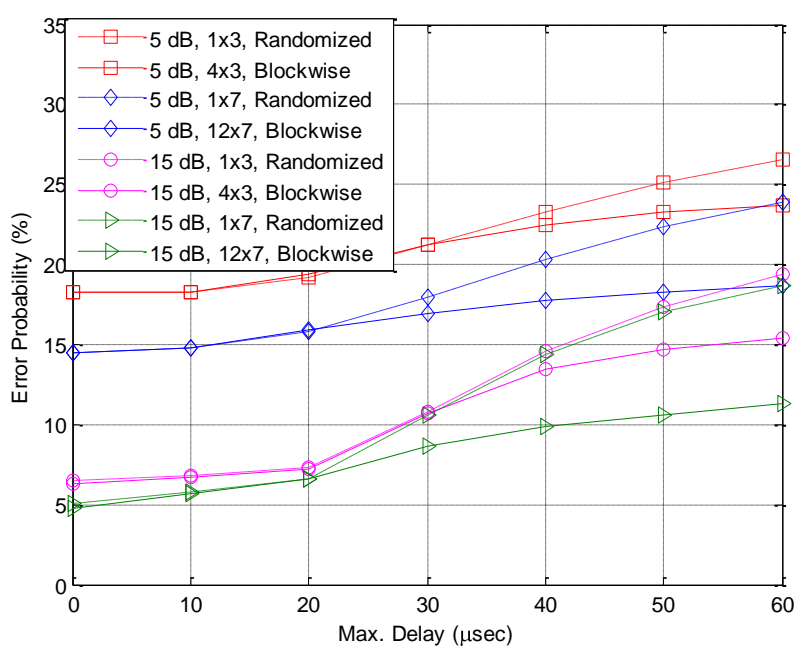

Fig. 7 Error probability versus $\tau_{\max }$ for energy detection with blockwise and randomized assignments.

Fig. 7 demonstrates the related simulation results, where the signals of all 12 users are considered to have the same power. An optimum energy detection threshold is used in all cases. It is observed that in randomized assignment, ICI has a more destructive effect on the detection performance. This is because in randomized assignment, each occupied subcarrier affects its adjacent subcarriers, some of which may be unoccupied. In the blockwise assignment, however, the subcarriers that are strongly affected are limited to the ones that are adjacent to each block. The two reasons for the error rates being higher for block size $4 \times 3$ compared to block size $12 \times 7$ are that number of subcarriers is smaller leading to a higher number of affected empty subcarriers, and number of symbols is smaller resulting in worse noise averaging.

\section{CONCLUdING REMARKS}

In this paper, a framework for an OFDMA based co-channel femtocell implementation is provided for next-generation broadband wireless communication systems. Co-channel interference is avoided by determining the spectra of closely located users and not using their resource blocks both in the UL and DL. Moreover, the impact of ICI is taken into account, which occurs due to asynchronous arrivals of mMS signals to an $\mathrm{fBS}$. In order to improve the spectrum sensing results, the scheduling information obtained from the $\mathrm{mBS}$ is utilized. The proposed framework may help in solving the interference problems observed in the CSG mode of operation in OFDMA based femtocell networks, where, a macrocell MS located close to a femtocell may cause significant interference to (and receive significant interference from) that femtocell.

\section{ACKNOWLEDGMENT}

The authors would like to thank Dr. Fujio Watanabe of DOCOMO Communications Labs, USA, for his helpful inputs.

\section{REFERENCES}

[1] V. Chandrasekhar, J. G. Andrews, and A. Gatherer, "Femtocell networks: a survey," IEEE Commun. Mag., vol. 46, no. 9, pp. 59-67, Sep. 2008.

[2] S. P. Yeh, S. Talwar, S. C. Lee, and H. Kim;, "WiMAX femtocells: a perspective on network architecture, capacity, and coverage," IEEE Commun. Mag., vol. 46, no. 10, pp. 58-65, Oct. 2008.

[3] H. Claussen, L. Ho, and L. Samuel, "An overview of the femtocell concept,” Bell Labs Tech. J., vol. 13, no. 1, pp. 221-245, 2008.

[4] M. Rumney, "IMT-Advanced: 4G Wireless Takes Shape in an Olympic Year," White Paper, Sep. 2008. [Online]. Available: http://cp.literature.agilent.com/litweb/pdf/5989-9793EN.pdf

[5] J. Choi, D. Shin, and D. Shin, "Research and implementation of the context-aware middleware for controlling home appliances," IEEE Trans. Consum. Electron., vol. 51, no. 1, pp. 301-306, 2005.

[6] FemtoForum, "Femtocell applications." [Online]. Available: http://femtoforum.org/femto/applications.php

[7] "3rd Generation Partnership Project; Technical Specification Group Radio Access Networks; 3G Home NodeB Study Item Technical Report (Release 8)," 3GPP, 3GPP TR 25.820, March 2008.

[8] FemtoForum, "Interference management in UMTS femtocells," White Paper, Dec. 2008. [Online]. Available: http://www.femtoforum.org/femto/Files/File/Interference Management in UMTS Femtocells.pdf

[9] V. Chandrasekhar and J. G. Andrews, "Uplink capacity and interference avoidance for two-tier cellular networks," in Proc. IEEE Global Telecommun. Conf. (GLOBECOM), Washington, DC, Nov. 2007, pp. 3322-3326.

[10] S.-Y. Tu, K.-C. Chen, and R. Prasad, "Spectrum sensing of OFDMA systems for cognitive radios," in Proc. IEEE Int. Symp. Personal Indoor and Mobile Radio Commun. (PIMRC), Athens, Greece, Sep. 2007, pp. 1-5.

[11] F. S. Chu and K. C. Chen, "Radio resource allocation in OFDMA cognitive radio systems," in Proc. IEEE Int. Symp. Personal, Indoor and Mobile Radio Commun. (PIMRC), Athens, Greece, Sep. 2007, pp. $1-5$.

[12] T. H. Kim and T. J. Lee, "Spectrum allocation algorithms for uplink sub-carriers in OFDMA-based cognitive radio networks," in Proc. IEEE Int. Conf. on Innovations in Information Technol., Nashville, TN, Nov. 2007, pp. 51-54.

[13] D. L. Perez, A. Valcarce, G. D. L. Roche, E. Liu, and J. Zhang; "Access methods to WiMAX femtocells: A downlink system-level case study," in Proc. IEEE Int. Conf. Commun. Syst. (ICCS), Guangzhou, China, Nov. 2008, pp. 1657-1662.

[14] S. Chen and T. Yao, "Intercarrier interference suppression and channel estimation for OFDM systems in time-varying frequency-selective fading channels," IEEE Trans. Consum. Electron., vol. 50, no. 2, pp. 429-435, 2004.

[15] I. Guvenc, "Statistics of macrocell-synchronous femtocell-asynchronous users' delays for improved femtocell uplink receiver design," IEEE Commun. Lett., vol. 13, no. 4, pp. 239-241, Apr. 2009.

[16] H. Yoshino and A. Czylwik, "Adaptive co-channel interference (CCI) cancellation for OFDM communication systems," in Proc. Internat. Zurich Seminar Broadband Commun., 2000, pp. 245-250. 
[17] M. Sahin and H. Arslan, "MIMO-OFDMA Measurements; Reception, Testing, and Evaluation of WiMAX MIMO Signals With a Single Channel Receiver," IEEE Trans. Instr. Meas., vol. 58, no. 3, pp. 713721, Mar. 2009.

\section{BIOGRAPHIES}

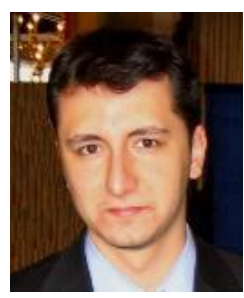

Mustafa Emin Sahin received his B.S. degree in Electrical and Electronic Engineering from Boğaziçi University, Istanbul, Turkey, in June 2004, and his M.S. degree in Electrical Engineering from the University of South Florida, Tampa, FL, USA, in May 2006. $\mathrm{He}$ is a member of the Wireless Communications and Signal Processing Group at University of South Florida and he is working toward his Ph.D. degree. During his Ph.D. study, he has been collaborating with researchers in DOCOMO USA Communications Laboratories, Palo Alto, CA. His research interests include OFDMA-based co-channel femtocells, co-channel interference cancellation in OFDMA, MIMO implementation in WiMAX systems, and spectrum sensing in cognitive radios.

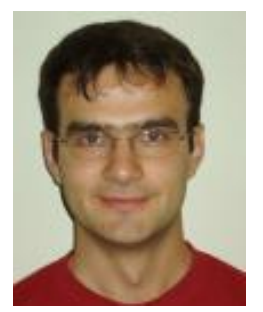

Ismail Guvenc (M'06) received his B.S. degree from Bilkent University, Turkey, in 2001, M.S. degree from University of New Mexico, Albuquerque, NM, in 2003, and $\mathrm{Ph} . \mathrm{D}$. degree from University of South Florida, Tampa, FL, in 2006 (with Outstanding Dissertation Award from USF Graduate School), all in Electrical Engineering. $\mathrm{He}$ was with Mitsubishi Electric Research Labs in Cambridge, MA, in 2005. Since June 2006, he has been with DOCOMO USA Labs, Palo Alto, CA, working as a research engineer. His recent research interests include femtocells, LTE systems, cognitive radio, and UWB communications and localization. He has published more than 50 international conference and journal papers, and several standardization contributions for the IEEE 802.15 and IEEE 802.16 standards. Dr. Guvenc has served in the organizing and technical program committees of several international conferences, and co-authored a book on ultrawideband position estimation. He has over 15 pending U.S. patent applications and he is a member of the IEEE.

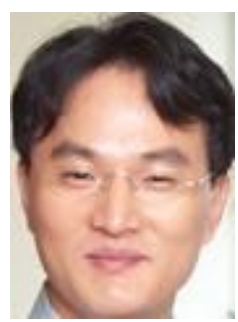

Moo Ryong Jeong (M'89) received B.S. and M.S. in Electronics Engineering from Seoul National University in 1991 and 1993. From 1994 to 1997, he worked as a patent attorney at Intellectual Property Group of KIM \& CHANG, the largest law firm in Korea. From 1997 to 2001, he studied at the University of Tokyo under the Japanese Government (Monbusho) Scholarship program. He received Ph.D. in Information and Communication Engineering at the same university in 2001. From 2001 to 2002, he was a research associate of the Dept. of Frontier Informatics, Graduate School of Frontier Sciences at the University of Tokyo. Since 2001, he has been working as a research engineer at DOCOMO USA Labs. He has been involved in the research and the standardization of packet based wireless system including 802.11 WiFi and 802.16 WiMAX. His current research interest is on the cellular systems for $4 \mathrm{G}$ and beyond. He published papers in the area of packet scheduling, wireless QoS management, 802.11 and 802.16 protocols, multi-hop relay, and femto-cell. He contributed many technical proposals to 802.11 and 802.16 standards. He co-authored a book on the next generation mobile system. He received the paper of the year award from IEICE in 2001 and the best paper award at Multimedia, Distributed, Cooperative and Mobile Symposium (DICOMO '99) in 1999. He has been a member of IEEE since 1989.

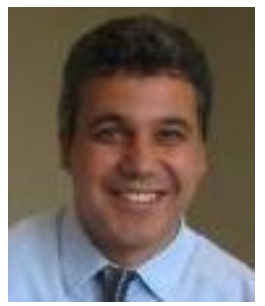

Dr. Huseyin Arslan (M'95-SM'03) has received his $\mathrm{PhD}$. degree in 1998 from Southern Methodist University (SMU), Dallas, Tx. From January 1998 to August 2002, he was with the research group of Ericsson Inc., NC, USA, where he was involved with several project related to $2 \mathrm{G}$ and $3 \mathrm{G}$ wireless cellular communication systems. Since August 2002, he has been with the Electrical Engineering Dept. of University of South Florida. In addition, he has worked as part time consultant for various companies and institutions including Anritsu Company, The Scientific and Technological Research Council of Turkey- TUBITAK, Lecroy, and XG technologies.

Dr. Arslan's research interests are related to advanced signal processing techniques at the physical layer, with cross-layer design for networking adaptivity and Quality of Service (QoS) control. He is interested in many forms of wireless technologies including cellular, wireless PAN/LAN/MANs, fixed wireless access, and specialized wireless data networks like wireless sensors networks and wireless telemetry. The current research interests are on UWB, OFDM based wireless technologies with emphasis on WIMAX and IMT-Advanced, and cognitive and software defined radio. He has served as technical program committee chair, technical program committee member, session and symposium organizer, and workshop chair in several IEEE conferences. He is a member of the editorial board for "Wireless Communication and Mobile Computing Journal" and "Research Letters in Communications". Dr. Arslan is a senior member of IEEE. 